\title{
Torta de buriti (Mauritia flexuosa) como ingrediente alternativo em rações para juvenis de Tambaqui (Colossoma macropomum)
}

\author{
Buriti pie (Mauritia flexuosa) as an alternative ingredient in rations for juveniles of Tambaqui \\ (Colossoma macropomum)
}

Ración de burití (Mauritia flexuosa) como ingrediente alternativo en las dietas para juveniles de Tambaqui (Colossoma macropomum)

\section{Resumo}

Neste trabalho estudou-se o efeito da inclusão de níveis crescentes de torta de buriti (Mauritia flexuosa) sobre os parâmetros produtivos e a viabilidade econômica. Neste experimento utilizou-se 360 peixes com peso médio inicial de 7,7 $\pm 2,8 \mathrm{~g}$ distribuídos em nove caixas de água com capacidade para 360 litros cada uma, em um delineamento experimental inteiramente casualizado, com três tratamentos de inclusão de farinha de torta de buriti $(0 ; 15 ; 30 \%)$ e três repetições. Em um período de 60 dias, foram mensurados os parâmetros físicos e químicos da água: temperatura, oxigênio dissolvido, $\mathrm{pH}$, amônia total, dureza e nitrito. E o desempenho zootécnico dos juvenis foi medido através dos índices de conversão alimentar aparente, peso final, taxa de crescimento específico, ganho de peso diário e sobrevivência. A viabilidade econômica foi determinada pela análise do custo médio do alimento por quilograma de peso vivo ganho. Os resultados foram submetidos a análises de variância (One way ANOVA) e as médias de cada tratamento comparadas pelo teste de Tukey, ao nível de 5\% de probabilidade. Os valores para as variáveis estudadas, não apresentaram diferença significativa entre os tratamentos, porém há uma tendência de aumento da taxa de conversão alimentar com o aumento da inclusão de torta de buriti. Concluiu-se que, com a metodologia utilizada, a inclusão da torta na dieta não reduziu os custos de produção e que é inviável a inclusão de torta de buriti na alimentação de juvenis de Tambaqui.

Palavras-chave: Alimento alternativo; Custos; Piscicultura; Produção.

\begin{abstract}
In this work, the effect of including increasing levels of buriti pie (Mauritia flexuosa) on production parameters and economic viability was studied. In this experiment 360 fish with an initial average weight of $7.7 \pm 2.8 \mathrm{~g}$ were distributed in nine water tanks with a capacity of 360 liters each, in a completely randomized experimental design, with three inclusion treatments of buriti pie flour $(0 ; 15 ; 30 \%)$ and three repetitions. Over a period of 60 days, the physical and chemical parameters of the water were measured: temperature, dissolved oxygen, $\mathrm{pH}$, total ammonia, hardness and nitrite. And the zootechnical performance of the juveniles was measured through the apparent feed conversion indices, final weight, specific growth rate, daily weight gain and survival. Economic viability was determined by analyzing the average cost of food per kilogram of live weight gained. The results were submitted to analysis of variance (One way ANOVA) and the means of each treatment compared by Tukey test, at the level of 5\% probability. The values for the studied variables did not show significant difference between the treatments, but there is a trend of worsening in feed conversion with the increase in the inclusion of buriti pie. It was concluded that the inclusion of the pie in the diet did not reduce the production costs and that the inclusion of buriti pie in the diet of Tambaqui juveniles is unfeasible.
\end{abstract}

Keywords: Alternative food; Costs; Fish farming; Production. 


\begin{abstract}
Resumen
En este trabajo se estudió el efecto de incluir niveles crecientes de pastel de burití (Mauritia flexuosa) sobre los parámetros de producción y la viabilidad económica. En este experimento se distribuyeron 360 peces con un peso promedio inicial de $7.7 \pm 2.8 \mathrm{~g}$ en nueve tanques de agua con una capacidad de 360 litros cada uno, en un diseño experimental completamente al azar, con tres tratamientos de inclusión de harina de burití $(0 ; 15 ; 30 \%)$ y tres repeticiones. Durante un período de 60 días, se midieron los parámetros físicos y químicos del agua: temperatura, oxígeno disuelto, $\mathrm{pH}$, amoníaco total, dureza y nitrito. $\mathrm{Y}$ el desempeño zootécnico de los juveniles se midió a través de los índices aparentes de conversión alimenticia, peso final, tasa de crecimiento específico, ganancia de peso diaria y supervivencia. La viabilidad económica se determinó analizando el costo promedio de los alimentos por kilogramo de peso vivo ganado. Los resultados se sometieron a análisis de varianza (ANOVA de una vía) y se compararon las medias de cada tratamiento mediante la prueba de Tukey, al nivel de $5 \%$ de probabilidad. Los valores de las variables estudiadas no mostraron diferencia significativa entre los tratamientos, pero existe una tendencia de empeoramiento en la conversión alimenticia con el aumento en la inclusión de harinha de burití. Se concluyó que con la metodología utilizada, la inclusión del harinha en la dieta no redujo los costos de producción y que la inclusión del harinha de burití en la dieta de los juveniles de Tambaqui es inviable.
\end{abstract}

Palabras clave: Alimento alternativo; Costos; Piscicultura; Producción.

\title{
1. Introdução
}

A Aquicultura é o cultivo de organismos cujo ciclo de vida em condições naturais, incide total ou parcialmente em meio aquático. Enquanto atividade econômica, encontra-se em plena expansão (FAO, 2020). É considerada uma atividade multidisciplinar, referente ao cultivo de diversos organismos aquáticos, incluídos neste contexto plantas aquáticas, moluscos, crustáceos e peixes, sendo que a intervenção ou manejo do processo de criação é imprescindível para o aumento da produção (Oliveira, 2009).

É inegável o potencial aquícola do Brasil, considerando seus recursos naturais propícios, espécies variadas e adequação climática, o que favorece o desenvolvimento das diversas atividades da aquicultura (Camargo \& Pouey, 2005). O país possui uma costa marítima com cerca de 8,5 mil km de extensão e uma Zona Econômica Exclusiva (ZEE) em torno de 4 milhões de $\mathrm{km}^{2}$, o representa quase metade de seu território. Além disso, detém aproximadamente 13,7\% do total de água doce do mundo são 10 milhões de hectares de lâmina d'água, terras alagadas, reservatórios e estuários, o que o coloca como a última grande fronteira da aquicultura no mundo. Porém, para que este potencial aquícola seja alcançado, diversos gargalos da produção necessitam ser solucionados, pois o crescimento da aquicultura brasileira não deve estar atrelado apenas às potencialidades naturais, uma vez que elas não podem, isoladamente, garantir o desenvolvimento da atividade (Ostrensky et al., 2008).

Sem dúvidas, um dos principais fatores que limitam a expansão da aquicultura é a formulação de rações balanceadas de baixo custo. A alimentação na piscicultura pode representar até $80 \%$ do custo de produção (Cheng et al., 2003). Entre as estratégias utilizadas para reduzir estes custos, a disponibilização de informações sobre as exigências nutricionais da espécie e a busca por alimentos alternativos são determinantes para a formulação de dietas específicas para diferentes fases do cultivo.

O Tambaqui (Colossoma macropomum) é um peixe originário da América do Sul, das bacias dos rios Amazonas e Orinoco (Gomes \& Araújo Lima, 2005). É o segundo maior peixe de escamas da Amazônia, atingindo mais de um metro de comprimento e $30 \mathrm{~kg}$ no ambiente natural (Araújo Lima \& Goulding, 1998). É onívoro e pode utilizar diferentes fontes proteicas e energéticas com eficiência numa ampla faixa de combinações entre elas (Oliveira et al., 2006). Seus dentes possuem formato molariforme multicúspide, adaptados a quebrar e esmagar frutos e sementes duras (Araújo Lima e Goulding, 1997; Goulding \& Carvalho, 1982).

É uma excelente opção para a piscicultura nacional, pois apresenta várias características favoráveis ao confinamento. É destacada a sua grande rusticidade, sendo bem adaptado a temperaturas entre $26^{\circ}$ e $35^{\circ} \mathrm{C}$ (Saint-Paul, 1986), com tolerância às baixas concentrações de oxigênio dissolvido na água, boa aceitação de subprodutos agroindustriais, boa conversão alimentar, boa adaptação ao cultivo,com crescimento relativamente rápido (Graef, 1995). Ressalta-se ainda a facilidade de manejo dessa espécie para reprodução induzida, com consequente disponibilidade de juvenis praticamente o ano inteiro (Castagnolli, 1992). 
Essas características lhe permitem lugar de destaque no cenário aquícola nacional, sendo a espécie nativa que mais se cultiva e a segunda entre todas as espécies cultivadas (IBGE, 2020; PEIXES BR, 2021).

A viabilidade econômica da criação de tambaqui, como em qualquer outra espécie animal, depende da nutrição. Apesar das características zootécnicas favoráveis, o custo com alimentação do tambaqui é considerado alto, representando cerca de 60$70 \%$ dos custos de produção (Rotta, 2002). Dessa forma, faz-se necessário a determinação das exigências nutricionais, a busca por alimentos alternativos e econômicos e a formulação de rações completas para o sucesso e sustentabilidade da produção, em especial do Tambaqui (Rotta, 2002).

O buritizeiro (Mauritia flexuosa) é uma palmeira da família Arecaceae, encontrada nos estados do Pará, Amazonas, Amapá, Rondônia, Goiás, Bahia, Minas Gerais, Mato Grosso, Ceará, Maranhão, Piauí e Tocantins (Manhães, 2007; Souza, 2011). É um fruto sazonal, onde sua frutificação em maior escala ocorre nos meses de dezembro a junho na maioria das regiões (Leão, 2005). A maturação dos frutos pode ser bem heterogênea dentro de um mesmo buritizal, variando de 7 a 11 meses. Seu fruto é uma drupa globoso-alongada de 4-7 cm de comprimento, constituída de epicarpo formado de escamas rombóides, de cor castanho-avermelhada, mesocarpo representado por uma massa espessa de cor alaranjada e endocarpo esponjoso que envolve a semente (Gazel-Filho \& Lima, 2001; Lorenzi et al., 2006).

A polpa de buriti contém quantidades consideráveis de carotenóides, polifenóis e ácido ascórbico, podendo ser usada na prevenção de inúmeras doenças advindas do estresse oxidativo, sendo sua quantidade de $\beta$-caroteno superior ao encontrado na couve e cenoura (Manhães, 2007).

Sua fração lipídica é composta basicamente de tocoferol e óleos com predominância dos ácidos graxos oléico, palmítico e ômega-9, que auxiliam na prevenção de doenças cardiovasculares (Manhães, 2007). Possui grandes quantidades de aminoácidos sulfurados importantes para bebês prematuros e triptofano precursor de niacina, sendo também rica em fibras, além de dispor da presença de diversos minerais (Manhães, 2007).

Neste sentido, esta pesquisa teve como objetivo avaliar a viabilidade econômica da ração alternativa com níveis crescentes de inclusão de polpa de buriti, em substituição ao farelo de milho, na alimentação de juvenis de Tambaqui.

\section{Metodologia}

O experimento teve duração de 60 dias e contou com meio milheiro de alevinos de Tambaqui com peso médio de meio grama $(0,5 \mathrm{~g})$, proveniente de um produtor particular próximo à cidade de Castanhal - PA. Os alevinos foram aclimatados em um tanque de alvenaria e lá permaneceram por 14 dias, alimentados com ração comercial contendo $36 \%$ de proteína bruta até que atingissem peso médio de $15 \mathrm{~g}$, peso de início do experimento.

Para elaboração do alimento alternativo, foi utilizada polpa de buriti, adquirida junto à Cooperativa Agroextrativista do Rio Pagão (COPA) do município de Curralinho, arquipélago do Marajó - PA. Por se tratar de um produto úmido, a polpa foi desidratada ao sol, por 48 horas, sendo posteriormente moída em moinho tipo martelo, com matriz fina de 0,8 milímetros. Para uniformização das partículas, todos os outros ingredientes utilizados na composição das rações - farelo de soja, farelo de milho, farelo de trigo - passaram pelo mesmo processo de moagem. Além destes ingredientes, utilizou-se na formulação das rações, óleo de soja e premix polivitamínico. As rações foram formuladas de modo a serem isoprotéicas (28\% PB), com níveis crescentes de inclusão de farinha de polpa de buriti $(0,15$, e $30 \%)$.

Os grupos testados foram denominados como: I, II e III, respectivamente. Para formulação das rações experimentais, foi utilizado o programa Microsoft Excel 2010. No preparo das dietas experimentais, todos os ingredientes foram misturados e processados em máquina bivolt moedora de carne modelo C.A.F-05, com matriz de $4 \mathrm{~mm}$ de diâmetro para a formação dos grânulos. Para secagem, foi utilizada lona plástica preta, onde foram colocadas as rações sob o sol, por um período de 5 horas, até ficarem bem secas, evitando assim a proliferação de fungos. Em seguida, foram acondicionadas em sacos plásticos de $15 \mathrm{~kg}$ 
e mantidas em temperatura ambiente. Os níveis de inclusão dos ingredientes em cada ração experimental estão evidenciados na Tabela 1 .

Tabela 1. Composição percentual das dietas experimentais com níveis crescentes de inclusão de farinha de polpa de buriti.

\begin{tabular}{lccc}
\hline \multirow{2}{*}{ INGREDIENTES } & \multicolumn{3}{c}{ Dieta experimental em \% / 1Kg } \\
\cline { 2 - 4 } & Controle & $15 \%$ & $30 \%$ \\
\hline Farelo de soja & 330 & 360 & 400 \\
Farelo de trigo & 320 & 280 & 170 \\
Farelo de milho & 320 & 180 & 100 \\
Farelo de buriti & 0 & 150 & 300 \\
Óleo de soja & 20 & 20 & 20 \\
Premix vitamínico & 10 & 10 & 10 \\
\hline Total & $\mathbf{1 0 0 0}$ & $\mathbf{1 0 0 0}$ & $\mathbf{1 0 0 0}$ \\
\hline
\end{tabular}

Fonte: Autores.

\subsection{Análises químico-bromatológica}

As análises de composição centesimal das dietas contendo níveis crescentes de inclusão de torta de buriti foram realizadas seguindo a metodologia descrita pela A.O.A.C (1995).

\subsection{Protocolo experimental}

O experimento foi realizado em nove caixas d'água com volume útil de 360 litros, abastecidos com água de poço artesiano, com taxa de renovação diária de $10 \%$ do volume total, aeração constante e cobertas com tela 12 mm para evitar perdas dos animais. Foram realizados três tratamentos com três repetições, de acordo com os diferentes níveis de inclusão da farinha de buriti, sendo estes de 0,15 e 30\%. O grupo controle foi o tratamento em que não houve inclusão de farinha de polpa de buriti. Foram alocados quarenta juvenis de Tambaqui, de aproximadamente 7,7 $\pm 2,8 \mathrm{~g}$ por unidade experimental, em um delineamento inteiramente casualizado (Tabela 2).

Tabela 2. Disposição dos diferentes tratamentos e seus respectivos níveis de inclusão de torta de buriti.

\begin{tabular}{|c|c|c|c|c|c|c|c|c|c|}
\hline Caixas d'água & $\mathrm{I}$ & II & III & IV & $\mathrm{V}$ & VI & VII & VIII & IX \\
\hline Níveis de inclusão & $30 \%$ & $30 \%$ & $0 \%$ & $15 \%$ & $0 \%$ & $15 \%$ & $15 \%$ & $30 \%$ & $0 \%$ \\
\hline
\end{tabular}

Fonte: Autores.

Após um período de adaptação às condições experimentais de setes dias, as unidades foram alimentadas com ração comercial contendo $36 \%$ PB. Os animais passaram por um jejum de 24 horas para, posteriormente, serem alimentados duas vezes ao dia (às 7:30 e 17:30h) com as rações testadas, ao nível de 5\% da biomassa.

\subsection{Parâmetros físicos e químicos da água}

Durante o período experimental foram aferidos os parâmetros de qualidade da água, sendo realizadas a cada três dias, medidas de amônia ([média \pm desvio]), nitrito ([média \pm desvio]), através dos métodos colorimétricos; e dureza ([média \pm desvio]), por meio de titulação. Também a cada três dias, os teores de oxigênio dissolvido foram avaliados através de um oxímetro digital e as medidas de temperatura ([média \pm desvio]) $\mathrm{pH}$ ([média \pm desvio]) e condutividade ([média \pm desvio]) foram aferidas com uma sonda multiparâmetro digital. 


\subsection{Biometria}

Ao longo do experimento foram realizadas três biometrias, uma realizada no início do experimento e as demais em um intervalo de vinte dias. Estas últimas possibilitaram avaliar o efeito de cada ração sobre o desempenho dos peixes. Para reduzir os efeitos de estresse durante a manipulação dos indivíduos, foi utilizado anestésico benzocaína a $100 \mathrm{mg}$ L-1 de água (Gomes et al., 2001).

\subsection{Análise estatística}

Para avaliar a influência da substituição do milho pela farinha de buriti no custo produtivo, foi determinado o custo médio do alimento por quilograma de peso vivo ganho, segundo (Bellaver et al., 1985). Para avaliar os efeitos dos tratamentos foi usado o teste de Cochran ao nível de 5\% de significância. Os valores dos parâmetros de desempenho zootécnico dos juvenis foram submetidos à análise de variância (One-way ANOVA). As médias de cada tratamento foram comparadas pelo teste de Tukey, ao nível de 5\% de probabilidade. As análises foram efetuadas com o auxílio do programa Microsoft Excel 2019 e Statistica 7.0.

\section{Resultados e Discussão}

\subsection{Bromatologia das dietas experimentais e viabilidade econômica}

Os dados das análises da composição centesimal de cada ração experimental estão descritos na Tabela 3.

Tabela 3. Bromatologia das rações experimentais com diferentes níveis de inclusão de torta buriti utilizadas no experimento em juvenis de Tambaqui.

\begin{tabular}{lccc}
\hline \multirow{2}{*}{ Dietas } & \multicolumn{3}{c}{ Porcentagem de nutrientes nas dietas experimentais } \\
\cline { 2 - 4 } & UM & CZ & PB \\
\hline Controle & 8,3 & 5,2 & 27,8 \\
$15 \%$ & 8,2 & 4,8 & 28,6 \\
$30 \%$ & 8,3 & 5,1 & 27,2 \\
\hline
\end{tabular}

$\mathrm{UM}=$ umidade; $\mathrm{CZ}=$ cinza; $\mathrm{PB}=$ proteína bruta. Fonte: Autores.

\subsection{Variáveis de desempenho produtivo}

As dietas contendo diferentes níveis de inclusão de farinha de polpa de buriti não apresentaram diferenças estatísticas significativas ao final do experimento, para as variáveis de desempenho produtivo analisadas (Tabela 4).

Tabela 4. Valores médios e desvio padrão do desempenho produtivo de juvenis de Tambaqui alimentados com rações contendo níveis crescentes de inclusão de torta de buriti.

\begin{tabular}{lcccccc}
\hline \multirow{2}{*}{ Níveis de Inclusão (\%) } & \multicolumn{7}{c}{ Parâmetros analisados } \\
\cline { 2 - 7 } & GP & CAA & GPD & TCE & TCR & S \\
\hline Controle & $9,8 \pm 1,2^{\mathrm{a}}$ & $3,9 \pm 0,5^{\mathrm{a}}$ & $0,1 \pm 0,0^{\mathrm{a}}$ & $16,3 \pm 2,0^{\mathrm{a}}$ & $127,2 \pm 16,2^{\mathrm{a}}$ & 100 \\
$\mathbf{1 5 \%}$ & $10,4 \pm 0,8^{\mathrm{a}}$ & $3,3 \pm 0,0^{\mathrm{a}}$ & $0,1 \pm 0,0^{\mathrm{a}}$ & $17,3 \pm 1,3^{\mathrm{a}}$ & $135,3 \pm 10,8^{\mathrm{a}}$ & 100 \\
$\mathbf{3 0 \%}$ & $8,0 \pm 0,9^{\mathrm{a}}$ & $4,7 \pm 0,5^{\mathrm{a}}$ & $0,1 \pm 0,1^{\mathrm{a}}$ & $13,4 \pm 1,6^{\mathrm{a}}$ & $105,3 \pm 12,6^{\mathrm{a}}$ & 100 \\
\hline
\end{tabular}

GP = Ganho de peso $(\mathrm{g}) ; \mathrm{CAA}=$ Conversão alimentar aparente; GPD = Ganho de peso diário (g); TCE = Taxa de crescimento específico $(\%)$; Taxa de crescimento relativo (\%); $\mathrm{S}=$ Sobrevivência (\%). Fonte: Autores. 
Observou-se uma tendência de aumento na taxa de Conversão Alimentar e uma diminuição do Ganho de Peso, quando se aumenta o nível de inclusão da farinha de buriti, porém não ocorreu mortalidade em nenhum dos tratamentos.

O ganho de peso diário permaneceu em $0,1 \pm 0,0 \mathrm{~g}$ para peixes alimentados com dieta controle e $15 \%$ de inclusão e 0,1 $\pm 0,1 \mathrm{~g}$ para peixes alimentados $30 \%$ de inclusão do produto alternativo. As dietas contendo diferentes níveis de inclusão de torta de polpa de buriti não apresentaram diferenças estatísticas significativas ao final do experimento para todas as variáveis de desempenho produtivo analisadas, mas evidenciam uma tendência de piora nas variáveis mensuradas.

Em uma pesquisa realizada para desenvolvimento de uma ração alternativa com base em baru, amêndoa originária de uma leguminosa arbórea, avaliou-se sua influência sobre os parâmetros zootécnicos da Tilápia-do-Nilo (Oreochromis niloticus). Braz (2021) testou rações com diferentes concentrações de baru e verificou que não há interferência no desempenho zootécnico, morfometria e qualidade nutricional dos peixes. No entanto, a autora demonstrou que ração com adição de 10\% de baru parece influenciar negativamente no crescimento, levando a uma heterogeneidade em relação à massa corporal. Já para a ração de 5\% não se observou esse crescimento desigual, melhorando a altura das vilosidades intestinais, sendo, portanto, a mais recomendada (Braz, 2021).

Outro trabalho testando a inclusão de alimento alternativo para juvenis de Tambaqui, avaliou como a introdução de níveis crescentes de camu camu (Myrciaria dubia), uma fruta amazônica, influenciava em parâmetros fisiológicos e zootécnicos em diferentes tratamentos. Aride et al. (2018) reportaram que os peixes alimentados com 15\% de camu camu ganharam mais peso e com 30\% melhoraram o desempenho natatório. Para concentrações acima disso, os autores relatam perda de nutrientes, o que levou a depreciação do desempenho produtivo e assimilação nutricional (Aride et al., 2018).

$\mathrm{O}$ farelo de dendê (Elaeis guineenses) também foi um alimento alternativo testado para Tambaqui, nesse caso, como substituto à farinha de milho (Zea mays). O estudo evidenciou que o farelo de dendê pode substituir até $25 \%$ do milho na dieta do Tambaqui, pois mantém o equilíbrio no desempenho econômico e zootécnico da produção (da Silva et al., 2020). Através dos resultados de desempenho produtivo dos animais foi possível analisar a viabilidade econômica (Tabela 5).

Tabela 5. Relação entre custo médio da ração por quilograma ganho no i-ésimo tratamento, pela quantidade média de ração consumida no i-ésimo tratamento, preço médio por quilograma de ração utilizada no i-ésimo tratamento, e ganho médio de peso do i-ésimo tratamento.

\begin{tabular}{lccccc}
\hline Ingredientes & Unidade & Valor Unitario R\$ & Controle (0\%) & $\mathbf{1 5 \%}$ & $\mathbf{3 0 \%}$ \\
\hline Farelo de milho & $\mathrm{kg}$ & 0,38 & 0,12 & 0,69 & 0,38 \\
Farelo de soja & $\mathrm{kg}$ & 0,89 & 0,30 & 0,32 & 0,58 \\
Farelo de trigo & $\mathrm{kg}$ & 0,45 & 0,14 & 0,26 & 0,77 \\
Óleo de soja & litro & 2,74 & 0,06 & 0,06 & 0,06 \\
Farelo de buriti & $\mathrm{kg}$ & 2,5 & 0,00 & 0,75 & 0,75 \\
Premix vitamínico & $\mathrm{kg}$ & 27,00 & 0,27 & 0,27 & 0,27 \\
Custo/kg de ração & - & - & 0,59 & 0,25 & 1,56 \\
Custo médio/kg/PV ganho & - & - & 0,94 & 1,41 & 2,41 \\
\hline
\end{tabular}

Fonte: Autores.

As dietas experimentais $0 \%, 15 \%$ e 30\% apresentaram os seguintes custos respectivamente; $\mathrm{R} \$ 0,94 / \mathrm{kg}, \mathrm{R} \$ 1,41 / \mathrm{kg}$ e $\mathrm{R} \$ 2,41 / \mathrm{kg}$, não apresentando, desta forma, uma redução nos custos de produção. Este resultado concorda com o obtido por Soares et al. (1998) para carpa capim Ctenopharyngodon idella, e por Galdioli et al. (2002) para curimbatá Prochilodus lineatus, que não verificaram diminuição dos custos de produção com níveis crescentes de inclusão de farelo de canola nas rações experimentais. Entretanto, discorda com os resultados encontrados por Chabalin et al. (1992), que utilizaram resíduos 
hortifrutigranjeiros na alimentação do pacu (Piaractus mesopotamicus) uma espécie que tem necessidade nutricional semelhante ao Tambaqui. A fim de avaliar a influência desse produto na redução do custo de produção, os autores observaram uma redução de 55\% no custo em peixes submetidos a tratamento em sistema de gaiolas flutuantes de $8 \mathrm{~m}^{3}$ de volume. Da Silva et al. (2020) também verificaram, a partir dos indicadores econômicos avaliados, que uma substituição de $25 \%$ do milho por farelo de dendê é viável na alimentação de Tambaqui, principalmente considerando que em determinadas épocas do ano o milho tem aumento de preço.

Os ingredientes de origem vegetal utilizados na alimentação dos peixes, como sementes de leguminosas, tortas de sementes oleaginosas e tubérculos, entre outros, são limitados devido à presença de grande variedade de substâncias antinutricionais. A presença destes fatores no alimento pode resultar em crescimento e conversão alimentar reduzidos (Francis et al., 2001). No presente estudo, a presença de alterações significativas das variáveis de desempenho produtivo sugere que a torta de polpa de buriti possui quantidade de fatores antinutricionais, de maneira que afeta os índices testados.

\section{Conclusão}

Baseado nos resultados encontrados, a inclusão de torta de buriti na dieta, com a metodologia utilizada, não apresenta redução nos gastos de produção, desta forma é inviável sua utilização. Sugerimos que pesquisas que venham a utilizar o buriti avaliem tratamentos que sejam capazes de eliminar fatores antinutricionais, que possivelmente possam ter influenciado nos resultados desta pesquisa, para então ser testado em alimentação de juvenis de Tambaqui.

\section{Referências}

Araújo-Lima, C. A. R. M. \& Gomes, L. C. (2005). Criação de tambaqui. In: Baldisserotto, B. \& Gomes, L. C. (Org). Espécies nativas para piscicultura no Brasil. UFSM. 175-202.

Araújo-Lima, C. \& Goulding, M. (1998). Os frutos do tambaqui: ecologia, conservação e cultivo na Amazônia. Tefé, AM: Sociedade Civil Mamirauá /CNPq, 187

Aride, P. H. R., Oliveira, A. M., Batista, R. B., Ferreira, M. S., Pantoja-Lima, J., Ladislau, D. S., \& Oliveira, A. T. (2018). Alterações em parâmetros fisiológicos de tambaqui (Colossoma macropomum) alimentados com dietas suplementadas com fruta amazônica camu camu (Myrciaria dubia). Brazilian Journal of Biology, 78(2), 360-367.

Batista, V. S. \& Petrere, Jr. M. (2003). Characterization of the commercial fish production landed at Manaus, Amazonas State, Brazil.Acta Amazonica 33(1): $53-65$.

Bellaver, C. Fialho, E. T. \& Protas, J. F. S. (1985). Radícula de malte na alimentação de suínos em crescimento e terminação. Pesquisa Agropecuária Brasileira, 20. 969-974.

Braz, A. J. (2021). Desenvolvimento E Utilização De Ração Para Tilápias-Do-Nilo A Base De Fruto Nativo Do Cerrado. Dissertação de Mestrado. Programa de Pós-Graduação em Conservação de Recursos Naturais do Cerrado. Instituto Federal Goiano. 40 p.

Camargo, S. D., \& Pouey, J. L. O. F. (2005). Aquicultura-um mercado em expansão. Revista Brasileira de Agrociência, 11(4), $393-396$.

Castagnolli, N. (1992). Criação de peixes de água doce. FUNEP.189p.

Chabalin, E., Palhares, F. J. V., Ferraz, J. A., \& Neves, E. M. (1992). Viabilidade econômica da utilização de resíduos hortifrutigranjeiros na criação do pacu, Piaractus mesopotamicus, em gaiolas. Boletim técnico do CEPTA, 5, 23-29.

Cheng, Z. J., Hardy, R. W., \& Usry, J. L. (2003). Effects of lysine supplementation in plant protein-based diets on the performance of rainbow trout (Oncorhynchus mykiss) and apparent digestibility coefficients of nutrients. Aquaculture, 215(1-4), 255-265.

da Silva, R. S., Lopes, J. R. T., do Espírito Santo, R. V., dos Santos, M. A. S., Cordeiro, C. A. M., Yoshioka, E. T. O., \& de Lourenço Júnior, J. B. (2020). Palm kernel meal (Elaeis guineensis) as a substitute for corn (Zea mays) in diets of Tambaqui (Colossoma macropomum). Aquaculture Research, 51(8), 3358-3366.

FAO - Food and Agricultural Organization. FAO (2020). Yearbook of Fishery and Aquaculture Statistics 2019.

Francis, G., Makkar, H. P. S. \& Becker, K. (2001). Antinutritional factors present in plant-derived alternate fish feed ingredients and their effects in fish. Aquaculture, 199(3-4):197-227. doi: https://doi.org/10.1016/s0044-8486(01)00526-9

Galdioli, E. M., Hayashi, C., \& Soares, C. M. (2002) Substituição da proteína do farelo de soja pela proteína do farelo de canola em rações para alevinos de curimbatá (Prochilodus lineatus V.). Revista Brasileira de Zootecnia 31. 552-559. 
Research, Society and Development, v. 10, n. 8, e24510817345, 2021

(CC BY 4.0) | ISSN 2525-3409 | DOI: http://dx.doi.org/10.33448/rsd-v10i8.17345

Gazel-Filho, A. B. G., Lima, J. A. S. (2001). O Buritizeiro (Mauritia flexuosa) e seu potencial de utilização. EMBRAPA.

Gomes, L. C., Chippari-Gomes, A. R., Lopes, N. P., Roubach, R. \& Araujo-Lima, C. A. R. (2001). Efficacy of benzocaine as an anesthetic in juvenile tambaqui Colossoma macropomum. Journal of the World Aquaculture Society, 32, 426-31

Graef, E. W. (1995). As espécies de peixes com potencial para criação no Amazonas. In: Val, A. L. \& Honczaryk, A. (Eds.). Criando peixes na Amazônia Manaus: INPA, p.29-43.

IBGE - Instituto Brasileiro de Geografia e Estatística. Produção da Pecuária municipal, Anual. https://www.ibge.gov.br/estatisticas/economicas/agricultura-epecuaria/9107-producao-da-pecuaria-municipal.html?=\&t=o-que-e

Leão, M. M., Carvalho, M. de F. C. C. (2005). Tabela Brasileira de Composição de Alimentos: uma contribuição do setor saúde para a promoção da segurança alimentar e nutricional no Brasil. In: Salay, E. (Org.). Composição de Alimentos: uma abordagem multidisciplinar. Campinas -SP: Núcleo de Estudos e Pesquisas em Alimentação - NEPA, 13-23.

Manhães, L. R. T. (2007) Caracterização da polpa de buriti (Mauritia flexuosa, Mart.): um potente alimento funcional. Universidade Federal Rural do Rio de Janeiro. 78p. Dissertação Mestrado.

Oliveira, R. C. O. (2009). Panorama da aqüicultura no Brasil: A prática com foco na sustentabilidade. Intertox de Toxicologia, Risco Ambiental e Sociedade, São Paulo, 2-78,

Ostrensky, A., Borghetti, J. R., \& Soto, D., (2008). Aquicultura no Brasil: o desafio de crescer.

Peixe BR - Associação Brasileira da Piscicultura. (2021). Anual. https://www.peixebr.com.br/anuario-2021/

Rotta, M. A. (2002) Use of energy and protein for fish, Corumbá: Embrapa Pantanal. 24p

Saint-Paul, U. (1986). Potential for aquaculture of South American fresh water fishes: a review. Aquaculture, Baton Rouge, 54. $205-240$.

Soares, C. M., Hayashi, C., \& Furuya, V. R. B. (1998). Farelo de canola na alimentação de alevinos de carpa capim (Ctenopharyngodon idella). Acta Scientiarum 20.395-400.

Sousa, E. L. C., Moraes, E. C. \& Carvalho, J. E. U. (2004). Biometria do fruto e germinação de sementes de buritizeiro (Mauritia flexuosa). http://ainfo. nptia.embrapa.br/digital/bitstream/item/44670/1/EVERTONCANUTO.pdf> 